\title{
Assessment of Knowledge and Practice on Breast Feeding Among Mothers of Children Aged Less Than Two Years and Associated Factors in Areka Town, Wolita Zone, Southern Ethiopia, 2018
}

\author{
Tomas Yeheyis (BSc, MSc) \\ Lecturer, College of medicine and health sciences, Hawasa University
}

\begin{abstract}
World health organization guideline recommend that continuous frequent and on-demand breast feeding until two years of age and beyond is essential for the child as well as the mother. Breast feeding aids general health, growth and development of the children. This study will Asses knowledge and practice on breast feeding among mothers of children aged less than two years and associated factors in Araka town, Woliat zone,South Ethiopia community based cross sectional study was conduct among 286 mothers in Areka town on May 2018. By using simple random sampling techniquel kebele was selected from 4 kebeles of Areka town and lottery method was used to get required sample size. structured questionnaire was used for collecting data from respondents .The data was analyzed and processed by SPSS window version 18 and presented in the form of percentage, proportions, and narrative sand associations are identified. From the total of 274 mothers, $56.6 \%$ of the mothers have good knowledge about the benefits of breast-feeding $43.6 \%$ poor knowledge about child breast feeding. From the total of $274,132(48.2 \%)$ have good practice of breast feeding and $142(51.2 \%)$ mothers have poor practice of breast feeding. multivariate logistic regression analysis showed mothers of age $20-24$ and mothers of age 30-34 practiced appropriate breastfeeding less likely than mothers with age greater than 35 years of age. Mothers who attended higher education were more knowledgeable when compared to mothers not attended formal education. Mothers who are government employ are more likely know about importance of breast feed as compared to students. The result of this study demonstrated that more than half of mothers of children under two years of age have good knowledge about benefit of breast feeding and less than half of respondent have good practice of breast feeding.
\end{abstract}

Keywords: Breast feeding, knowledge, practice

DOI: $10.7176 / \mathrm{JHMN} / 88-01$

Publication date: April $30^{\text {th }} 2021$

\section{Background}

Over two-thirds deaths occurring worldwide during the first year of life children are often associated with inappropriate feeding practices, especially due to sub optimal breast feeding (9).

More than 10 million children under the age of five die each year; $41 \%$ of these deaths occur in sub-Saharan Africa and another $34 \%$ in South Asia and the major contributor to their death is poor breastfeeding practices (10). Suboptimal breastfeeding contributes for $45 \%$ of neonatal infectious deaths, $30 \%$ of diarrheal deaths and $18 \%$ of acute respiratory deaths among under five children in developing countries (11). In Ethiopia suboptimal breastfeeding practices are the major contributor to an estimated 70,000 infant deaths per year which is $24 \%$ of the total infant death annually which can be significantly prevented by nutrition interventions such as breastfeeding for two years or more (12). Globally, increasing optimal breastfeeding practices could save an estimated 1.5 million infant lives annually (13). Alive and Thrive initiative had tried to improve breast feeding practice in Ethiopia. The Ministry of Health of Ethiopia has also tried to enhance the practice of optimal breast feeding practice by developing training manuals and implementation guidelines on breastfeeding; and incorporated it to the primary health care in line with the health extension program but still the practice is far from the global recommendations (14).

All in all breastfeeding interventions have the potential to prevent $13 \%$ of all under five years of age deaths in developing areas of the world, ranking it as the most important preventative approach for saving the life of millions of children; out of this $23 \%$ of deaths are preventable as a result of continued breastfeeding in the $6-24^{+}$ months age group(6)

Demographic and health survey conducted in Addis Ababa shows, exclusive breast feeding rate in those less than 4 months of age was found to be $32 \%$. Timely complementary feeding rate in the age group 6-9 months was, however, found to be $57.4 \%$. Mothers who did not initiate breast-feeding at all were $2.8 \%$ about $44 \%$ of the infants were bottle-fed (7).There are many factors associated with the practice of breastfeeding including maternal socio demographic characteristics, psychosocial factors, and early exclusive breastfeeding experiences. These factors vary from country to country reflecting different influences due to the differences in various circumstances (8).

Therefore,this study was conducted to find out breastfeeding mothers adherence to global recommendations 
on optimal breastfeeding practices and to assess optimal breastfeeding knowledge and practice of mothers who have less than two years of age children as well factors associated with this in Araka town, Wolita zone, South Ethiopia.

\section{Methods and Materials}

Study setting, design, period

The study is conducted in Araka town, Wolita zone, South Ethiopia. Found $532 \mathrm{~km}$ southwest from Addis Ababa, the capital city of Ethiopia. The study was conducted on May 2018 using Community based cross-sectional study design.

\section{Sample size determination}

The sample size was estimated using a the single population proportion formula assuming prevalence for breastfeeding 77\% from research by Biruk T/Selassie(22), 95\% confidence level, 5\% margin of error.

$\mathrm{n}=\left(\underline{\left.\mathrm{Z}_{\mathbf{\alpha} / 2}\right)^{2} \mathrm{P}(1-\mathrm{P})}\right.$

$$
\mathrm{d}_{-}^{2}
$$

Where; $\mathrm{n}=$ sample size

$\mathrm{p}=$ proportion of mothers who are breast feeding up to 2 years of age and beyond

$1-p=$ proportion of mothers who are not breast feeding up to 2 years of age and beyond

$\mathrm{d}=$ margin of error $=0.05$

Confidence interval $(\mathrm{CI})$ at $95 \%=1.96$

Proportion $(\mathrm{p})=0.77$ and $1-\mathrm{p}=0.33$

Then substituting the value in the formula

$$
\frac{(1.96)^{2}(0.77)(0.23)}{0.05^{2}}=272
$$

After considering $5 \%$ non- response rate, the final sample size $=\mathbf{2 8 6}$

\section{Sampling procedure and data collection}

Simple random sampling technique was used in sampling the study subjects. Out of the total 4 kebeles, 1 of them was selected by lottery method. To get the individual sample units (subjects) at household level from the randomly selected Keble, the number of household with children less than two years were identified from health extension workers. After that we entered those house numbers into computer and generated the required sample size. If there were mothers with more than one children aged less than 2 years in one household the youngest child will be selected for the study. If mothers had twin children aged less than 2 years, one child was selected by lottery method.But in the absence of eligible respondent in a given household a substitution will be made by an individual in the next household.

interviewer administered semi structured questionnaires was used with modification from other research as presented in literature review and UNICEF the data was collected through interviewer administered quaternaries of the group member of the research. the questionnaire consist the socio-demographic characteristic, knowledge and practice of breast feeding , and associated factors.

\section{Data processing \& analysis}

After the data was collected, questionnaires were reviewed and organized by investigators. . Data was checked for completeness, consistency and accuracy. The data were entered after defining variables and analyzed by SPSS window version 18 and Binary Logistic regression was performed. COR (crude odds ratio) along with 95\% confidence interval was used to determine the existence of an association between independent and dependent variables. Then multivariate logistic regression was used to decrease the effect of confounding factors. Statistical significance was declared with p-value less than 0.05 for multivariable and 0.25 for bivariate logistic regressions. Finally, the result is presented using tables, texts and other pictorial representation.

\section{Data quality control}

Before the data collection, the data collectors made sure that they have a common understanding of the questionnaire and the questionnaire was translated to Amharic and back to English. After data collection, internal consistency was checked by cross checking the collected data within on every days of data collection.

\section{Ethical consideration}

Ethical clearance was obtained from AMU, college of health science, department of nursing. Permission was obtained from Areka woreda administrative office and informed consent was obtained from individual respondent. the respondents have been told know that they have the right to be involved or not to be involved in the study, and that noninvolvement otherwise will not affect the clinical care they might receive. 


\section{Results}

\section{Characteristics of the Respondents}

Out of the 286 questionnaires that were run, 274 questionnaires were completed making a response rate of $96 \%$.Of the total respondents in this study mothers in age group 20-24 years of age making $119(43.4 \%)$. From total of $274100(36.5 \%)$ children were in age group of $6-11$, more than half $138(50.4 \%)$ children were male.More than seventy eight percent $(78.1 \%)$ of the respondents were married. Regarding educational status of the respondents, $125(45.6 .0 \%)$ of the respondents had attended secondary and preparatory school. Of the total mothers interviewed, the majority $136(49.6 \%)$ of them were Protestant by religion and the majority of respondents $150(54.7 \%)$ were house wives. (Table1)

\section{Knowledge and practice of breast feeding}

Mothers were assessed whether they have adequate knowledge about the benefits of breastfeeding or not. Benefits of breast-feeding such as contraceptive effect, nutritious, protective and related importance were mentioned in different ways to ascertain the level of mothers' knowledge. In order to examine the knowledge of mothers about the benefits of breast-feeding, all there relevant variables were selected and considered as hundred percent, then positive responses were scored in respect to the number of participants. Finally, those positive responses for $75 \%$ and above of the relevant variables regarding to the benefits of breast-feeding, were labeled as satisfactory knowledge while for those less than $75 \%$, labeled as unsatisfactory knowledge. Based on this assumption, 155 (56.6\%) of the mothers have good knowledge about the benefits of breast feeding.

General practices such as breast-feeding styles (feeding on demand or program), exclusive breast feeding, frequency or intensity of breast-feeding, and related practices were included here. In order to explain the state of the general practices about breast-feeding all the relevant individual breastfeeding practices were considered as hundred percent. Finally, those optimal practices that have been practiced by the mothers for $75 \%$ and above of them, were labeled as good practice, while for those less than $75 \%$, labeled as "poor practice. Based on this assumption, around $133(48.2 \%)$ of the mothers have good practices of breastfeeding.

\section{Factors associated}

Factors associated with knowledge of breast feeding

The multivariate logistic regression analysis identified educational status of the mother, maternal employment, health educationas independent predictors of good knowledge for breast feeding.

Mothers who attended higher education were around 4 times more knowledgeable AOR: 4.866, $(, 0.904,26.175)$ at $(\mathrm{P}<0.031)$ when compared to mothers not attended formal education. Mothers who are government employ are 3.9 times more likely know about importance of breast feed as compared to students AOR 3.891 $(0.716,21.154)$ at $(\mathrm{P}<0.043)$. Mothers who have got satisfactory health education are 0.33 times less likely know about benefit of breast AOR 0.667(0.329, 1.350) $(\mathrm{P}<0.023)$.(Table 2)

\section{Factors associated with practice of breast feeding}

After adjustment for possible confounding variables for socio-demographic variables, multivariate logistic regression analysis showed mothers of age20-24 (AOR 0.51 [95\% CI 0.25, 1.010] and mothers of age 30-34 (AOR 0.58 [95\% CI 0.28, 1.105] were practiced appropriate breastfeeding less likely than mothers with age greater than 35 years of age. Likewise maternal occupation was significantly associated with breastfeeding practice. Mothers who are none governmental employ are 15 times have good practice AOR15.805(2.664, 93.763) as compared to students.

Other variables such as maternal age, number of party, and health education during ANC follow up, PNC visit and elsewhere didn't show any statistically significant association with optimal breastfeeding.(Table 3)

\section{Discussion}

This study assessed breastfeeding practices, knowledge and factors associated with breastfeeding in mothers who have less than two years children in the community of Areka SNNPR, Ethiopia. In this study, the majorities $(56.6 \%)$ of mothers have good knowledge about benefit of breastfeeding and less than half (48.2) of mothers have good practices on breast feeding. This result is higher than study in Jimma that is $28.9 \%$ and $28 \%$ knowledge and practice respectively (22). This might be due to currently in Ethiopia both urban and rural HEWs were trained by the help of NGO as well as governmental organization on area particularly optimal breastfeeding practices and how to increase maternal knowledge towards benefit of breast feeding were included like breastfeeding during maternal and child sickness and frequency of eating food during lactation.

Based on the findings from this study, maternal background characteristics such as occupation, attending formal education and attending health education have found to be more important for the mothers in acquiring knowledge about the benefits of breast-feeding and to practice appropriately.

Mothers who have got satisfactory health education are 0.33 times less likely know about benefit of breast 
AOR $0.667(0.329,1.350)(\mathrm{P}<0.023)$ as compared to those who do not get satisfactory health education. Mothers who have experienced these characteristics were found to be in favor of getting satisfactory knowledge about the benefits of breast-feeding. These findings are consistent with the study, about breast-feeding promotion in a diarrhea program, done in rural communities of Nigeria which showed seven days after the counseling, received individual focus breast-feeding counseling, more than half in the intervention group were found to be well oriented about the benefits of breast-feeding for their children $(\mathrm{p}<0.0001)$ as compared to the control groups. Another study on mothers' knowledge of preventive health, done in a slum area of Karachi, Pakistan, showed that mothers in the intervention group were more aware about the advantage of breast-feeding as compared to the non-intervention group $(\mathrm{p}<0.05)(18)$. Various studies have concluded that parenteral education especially that of mother is key element in improving children nutrition status.

Mothers who attended higher education were around 4 times more knowledgeable AOR: 4.866, $(, 0.904,26.175)(\mathrm{P}<0.031)$ when compared to mothers not attended formal education.A more educated mother likely to have higher income (which can indirectly affect her children health and nutrition) and higher status and power in the house hold as well as the community. This result shows that when mother education increases, mothers breastfeed their child optimally. This finding is consistent with study in Gujarat, India from July, 2008 to September, 2008 which showed paternal education has significant association with new-born exclusive breastfeeding [23]. The reason for this could be, if educational status of mother increases, the inter-spousal communication about breastfeeding increases and aware of benefit of encouraged breastfeeding practices

In this study occupational status was associated with the knowledge of the mothers. Mothers who are government employ are 3.9 times more likely know about importance of breast feed as compared to students AOR 3.891 $(0.716,21.154)(\mathrm{P}<0.043)$. Know a days there are different social interaction than previous that increase the knowledge of individuals and at the same time make difference on individual of especially different occupational status. Government employs could have better chance to have adequate information

Mothers of age 20-24 (AOR 0.51 [95\% CI 0.25, 1.010] and mothers of age 30-34 (AOR 0.58 [95\% CI 0.28, 1.105] were practiced appropriate breastfeeding less likely than that of mothers with age $>=35$.this may be due to as those mothers with age $>=35$ aremore experienced and mothers at age group from 20-24 and 30-34 are most of the time busy with work this indirectly decrease breast feeding practice. Likewise maternal occupation was significantly associated with breastfeeding practice. Mothers who are none governmental employ are 15 times have good practice AOR 15.805(2.664, 93.763) as compared to student. Employment of the mother may be expected to enhance accessibility of the household to income, which may in turn have a positive effect on the nutritional status of the child. This may be expected because such income is more likely to be controlled by the mother and used to improve children's nutritional status. However, it may also beargued that employment of the mother may have a negative effect on children'snutritional status by reducing both infants' access to breastfeeding and time spent on childcare. Some empirical studies show that the mothers of the most malnourished children work outside their home.

\title{
Conclusion
}

The result of this study demonstrated that more than half of mothers of children under two years of age have good knowledge about benefit of breast feeding and less than half of respondent have good practice of breast feeding. Regarding the factor associated with breast feeding practice, age of mother and maternal occupation significantly affect practice of breast feeding. Knowledge of mother significantly associated with educational status of mother, maternal occupation and health education during ANC follow up, PNC visit and elsewhere. But knowledge and practice of mother is not significantly associated with a marital status, ethnicity, monthly income, ANC follow up, PNC visit and place of child birth.

\author{
Abbreviations \\ AMU:-Arba Minch University \\ HSDP:-Health Services Development Program \\ NGO:-Non-Governmental Organization \\ SNNPR:-Southern Nation Nationality and People Region \\ UNICEF:-United Nations Children Fund \\ USA: - United States of America \\ WHO:-World Health Organization
}

\section{Acknowledgment}

The authors are great full to data collectors and study participants.

\section{REFERENCES}

1. WHO. Infant and young child feeding Model Chapter for textbooks for medical students and allied health 
professionals. Geneva, WHO; 2009

2. WHO. infant and young children breast feeding fact sheet N'342 February 2014

3. American academy of pediatrics section on breast feeding and the use of human milk" pediatrics $129(3) ; 827-842,2006$

4. WHO. Report of global consultation on summery of guiding principle for complementary feeding of breast fed child. WHO Geneva 2001

5. Federal ministry of health, national strategy for child survival Ethiopia ,edited by family health department, Addis Ababa Ethiopia 2005

6. World alliance of breast feeding .protecting, promoting and supporting continued breast feeding from 6-24 month. Global partners meeting vi Penda Malaysia 2008

7. Central Statistical Authority of Ethiopia and ORC Macro; Ethiopia Demographic and Health Survey. 2007

8. ] Li L, Zhang M. Factors associated with the initiation and duration of breastfeeding by Chinese mothers in Perth, Western Australia. J Hum Lact;20(2): 188-195, 2004

9. WHO. Global strategy for infant and young child feeding. Geneva:WHO;2003

10. Alive and thrive initiative, nurturing and grow. impact of early initiation of breast feeding on newborn death technical bruit issue 1,2010

11. WHO. Mortality and burden of disease attributable to selected major risks. Geneva: WHO; 2009.

12. Federal Ministry of Health. Ethiopian Health Sector Development Program IV: 2010/2011-2014/2015. Addis Ababa, Ethiopia; 2010.

13. Linkage feeding newborn and infant breast feeding. Anan's guideline to child care. Linkage academy for education development ,2006

14. Central Statistical Agency [Ethiopia] and ICF International. Ethiopia Demographic and health Survey .Addis Ababa, Ethiopia: 2012

15. M.C.Davit JA; et al; The impact of the health mass media campaign on timely initiation of breast-feeding in Jordan. Stud famePlan; 24 (5): 295-309; USA.1993

16. Yimyam S; Morrow M; Breastfeeding practices among employed Thai women in Chiang Mai. J. Hum Lact; 15 (3): 225-32; USA.2003

17. UNICEF-Assignment Children, A journal concerned with children, women and youth in development: Breast-feeding and health. 2nd edition;Geneva1981

18. Osman NA; el-sabban FF; Infant feeding practices in Al-Ain, United Arab Emirates. East Mediterranean health J; 5 (1): 103-10; Egypt 1999

19. Ababa b; Berhane Y; Weaning in Butajira, South Ethiopia: A study on mothers' knowledge and practice. Ethiopian Medical Journal; 36 (1): 37-45; Ethiopia.1998

20. Tema T; Knowledge, Attitude and practice towards exclusive breast-feeding in Jimma. Ethiopian J Health Science; 10 (1): 7-13; Ethiopia.2000

21. Birhanu T. Knowledge, Attitude and practice towards exclusive breast-feeding in Halaba. Ethiopian $\mathbf{J}$ Health Science; 10 (1): 7-13; Ethiopia.2000

22. Biruki T. Status of breast feeding on mothers of children under two years of age and implication for occurrence of acute diarrhea in jima southern 1, 2014.

23. Mahoney MC; James DM; Predictors of anticipated breast-feeding in an urban, low in come setting. J Fame Pract; 49 (6): 529-33; USA2008

24. Ketsela T, Kebede D; Pattern of feeding infants in Addis Ababa. Ethiop. J. Health Dev.; 10 (1): 57-651; Ethiopia1996

25. Bekele A, Magnitude and determinants of bottle-feeding in a peri-urban community: A Master thesis, Ethiopia. 2009

26. Bekele sh; Breast-feeding practices among factory working mothers and Housewives in Akaki Beseka. A master thesis paper. Ethiopia.

27. Benyamen; Y. S; Hassan, M. K. Feeding patterns in the first two years of life in Basra, Iraq. Eastern Mediterranean Health Journal; 4 (3): 448-451; Iraq 1998.

28. Eregie CO; Studies on exclusive breast-feeding: A report on associated factors in an African population. J Tropical Pediatrics; 44 (3): 172-3; 1998,

29. Davies-Adetugbo, Anita A; Scio-cultural factors and the promotion of breastfeeding in rural Yoruba communities of Osun, state, Nigeria. Social science and medicine; 45 (1): 113-125; Jul 1997,

30. Addis A; Socio - cultural factors related to breast-feeding in Jimma town: A thesis paper., A. Ababa1987

31. . Ketsela T, et al; Breast-feeding in Addis Ababa, Ethiopia: Results of a focus group study. Ethiop. J. Health Dev.; 10 (3): 133-143;Addis Ababa 2004.

32. WHO, Reading on diarrhea: student manual. Geneva 1998.

33. W Yimyam S; Morrow M; Breast- feeding practices among employed Thai women in Chiang Mai. J. Hum Lact; 15 (3): 225-32; 2003 Sep, USA HO, Reading on diarrhea: student manual. Geneva1998, 
34. Central Statistics Authority, Population Distribution of Ethiopia by Single Years of Age. 04-12-01, Addis Ababa, Ethiopia

35. Ketsela T; Asfaw M; Kebede D; Patter of breast-feeding in western Ethiopia and their relationship to acute diarrhea in infants. J Trop Pediatric; 36 (6): 180-3; 2008

36. UNICEF. The state of the world's children, focus on nutrition. Oxford University press 2003.

\section{Declarations}

\section{Ethics approval and consent to participant}

The study protocol was ethically approved by the Ethical Review Board (IRB) of Arbaminch University,College of medicine and Health Sciences. Official letter was written to Areka woreda Administrative office. The study posed a low or not more than a minimal risk to the study participants. The study did not involve any invasive procedures. Accordingly, after the objective of the study was explained, verbal consent was obtained from the respondents of the study. Moreover, the confidentiality of information was guaranteed by using code numbers rather than personal identifiers and by keeping the data locked.

Acknowledgements

The authors are grateful for the data collectors and study participants

Availability of data and material

The datasets used and/or analyzed during the current study is available upon request from the corresponding author and Co- authors.

\section{Consent for publication}

Not applicable

Author's contribution

SA,GW and BB conceived and designed the study and analyzed the data.. ZS and TY assisted with the design conception, advising and critically reviewed the manuscript. TY,AG,BW and AB prepared the manuscript. All authors have read and approved this manuscript.

Funding

There is no source of funding for this research. All costs were covered by researchers.

Competing interests

The authors declare that they have no competing interests. 